\title{
LA RECEPTION DU SURREALISME DANS LES REVUES LITTERAIRES D'AMERIQUE LATINE (1924-1950)
}

\author{
Claudine LECRIVAIN \\ Universidad de Cádiz.
}

Lorsque l'on aborde l'étude du surréalisme en Amérique Latine, on se demande souvent dans quelle mesure les auteurs étaient ou n'étaient pas surréalistes, quelles étaient leurs publications, comment pouvait se définir ce surréalisme, en quoi il pouvait être différent du mouvement français, mais on se pose rarement la question de savoir quels aspects du surréalisme français, quels auteurs furent connus dans le continent américain. Face à cette lacune notre intention est de dresser un bref bilan de la pénétration du surréalisme français en Amérique Latine, et dans le cadre de cet article nous aborderons exclusivement la présence dans les revues littéraires des auteurs qui participèrent au mouvement français. Il est certain que ce n'est là qu'un aspect de la diffusion, et qu'il serait nécessaire pour compléter cette brève étude de dresser le bilan des articles critiques afin d'analyser globalement la réception du surréalisme provenant de France. Les revues sont le véhicule le plus direct de la diffusion et la plupart des auteurs d'avant-garde se réunissent autour des revues littéraires qui se font écho de leurs aspirations et également des tendances extérieures, en particulier des courants littéraires en France, pays qui a longtemps exercé un grand attrait sur les artistes latino-américains. "A partir de la época de la independencia, los hispanoamericanos cultos tuvieron acceso por medio de revistas y periódicos a muchos escritos traducidos de autores extranjeros, franceses en su gran mayoría"1. Cet attrait ne cessa pas au XX siècle, même s'il s'amoindrit quelque peu par rapport aux époques antérieures.

Les courants littéraires européens en général, et français en particulier, étaient donc connus d'une majeure partie du public et des artistes. Il faut cependant remarquer qu'à partir de 1925, le commerce de librairie et d'éditions commença à s'émanciper de Paris, et aussi de Madrid, sans en arriver à rompre complètement les échanges. L'activité littéraire de l'Amérique Latine se voit donc marquée par cette connaissance de l'avant-garde européenne.

"Tristan Tzara, Paul Eluard, André Breton, Louis Aragon, Paul Morand, Blaise Cendrars, Drieu La Rochelle, Valéry Larbaud, Max Jacob fueron los mejores escritores conocidos de Hispanoamérica"2.

(1) Boyd G. Carter Historia de la literatura hispanoamericana a través de sus revistas. México. Ed de Andrea 1968 p. 16

(2) Anderson-Imbert, Enrique. Spanih American Literature. Vol. I. Detroit. Wayne State University Press. 1969. cité et traduit par Gerald J. Langowski El surrealismo en la ficción hispanoamericana. Madrid. Gredos. 1982. p. 24. 
Cependant il nous est impossible dans le cadre de cette analyse de considérer l'ensemble des pays de langue espagnole et nous n'en avons retenu que quatre (Argentine, Chili, Pérou et Mexique) qui nous semblent les plus représentatifs et que nous traiterons séparément partageant l'avis de Pierre Rivas "Les surréalismes latino-américains se développent entre 1926 et la seconde guerre indépendamment les uns des autres, selon une tradition d'isolement culturel interaméricain, chaque pays étant directement lié á l'épicentre parisien"3.

\section{Argentine}

Buenos Aires fut un centre important de l'avant-garde qui dans un premier temps put s'y épanouir grace à Jorge Luis Borges qui revint en 1921 après un séjour a Madrid et s'activa à introduire l'ultraisme, porteur d'une volonté de création originale et de rénovation de la poésie à l'intérieur de la communauté littéraire argentine dont bon nombre de poètes voyageaient fréquemment à Paris, Madrid et Barcelone.

Le véritable surréalisme argentin, c'est-à-dire un surréalisme exprimant une volonté de pénétrer la vie, d'explorer et de libérer toutes ses possibilités, ne prend forme qu'en 1928 lors de la création de la revue QUE par Aldo Pellegrini. "Yo conocía a Breton a través de la revista Littérature. El primer número de la revista La Révolution Surréaliste que llegó a mis manos a poco de su aparición me deslumbró. Comencé entonces a catequizar a algunos de mis compañeros estudiantes de medicina y en el año 1926 se fundó un grupo surrealista que comenzó a preparar una revista. Esta recien apareció en 1928 y un segundo número en 1930 con el nombre $Q U E " 4$. Mais nous ne sommes pas en mesure d'affirmer la présence d'auteurs français dans ces numéros, étant donné la difficulté d'accés à ces revues 5 .

Etrangement c'est dans la revue mensuelle $S U R$, publiée sous la direction de Victoria Ocampo, à partir de 1931, et qui n'était ni surréaliste, ni surréalisante, mais qui essayait de faire connaître de nombreux écrivains français (Valéry, Gide, Denis de Rougemont, Caillois, Bachelard..) que Breton publia "El castillo estrellado", essai poétique, qui parut dans Minotaure No 8 en 1936 et fut inclus postérieurement dans L'Amour fou.

Il faut attendre 1948 pour assister à la parution de Ciclo (deux numéros en 19481949): il ne s'agit pas d'une revue surréaliste, mais néanmoins René Char et André Breton y collaborèrent.

(3) Rivas, Pierre "Le surréalisme au Pérou" Mélusine No III Paris. L'Age d'Homme. 1982. p. 109-114.

(4) Baciu, Stefan. Antología de la poesía surrealista latinoamericana. Mexico. Joaquin Mortiz 1974

(5) Les collections sont malheureusement souvent incomplètes et les reproductions en facsimilé sont rares, à l'exception des revues mexicaines.

\section{Chili}

Vicente Huidobro qui eut de nombreux contacts avec la France, et qui d'ailleurs s'installa à Paris en 1916 oú il fonda la revue Nord-Sud avec Pierre Reverdy, et qui écrivait aussi bien en espagnol qu'en français ne semble pas avoir favorisé directement l'introduction du surréalisme. C'est au Chili que s'organisa une des expositions surréalistes, à laquelle participa Breton, Duchamp, Hérold, Magritte. Le catalogue de l'exposition comportait des textes de Teófilo Cid, Braulio Arenas, Breton et Péret. Cependant le groupe surréaliste chilien s'organisa autour de Cid, Arenas et Enrique Gómez-Correa qui fondérent la revue Mandrágora (Décembre 1938), mais ne publia ni textes ni poémes des surréalistes.

Puis parut la revue Leitmotiv en 1942 sous la direction de Braulio Arenas. On trouve dans le premier numéro la traduction de Prolégoménes à un troisième manifeste ou non de Breton et des poèmes de Benjamin Péret et Aimé Césaire. En 1949 Arenas publia un seul numéro de la revue Gradiva, où il inclut des textes de Gisèle Prassinos et Giorgio de Chirico. Là semble s'arrêter la diffusion du surréalisme français au Chili.

\section{Mexique}

Deux groupes importants marquèrent l'avant-garde au Mexique: tout d'abord l'estridentisme, fondé en 1923, qui lutta pour situer la littérature mexicaine sur le plan international de l'avant-garde littéraire et se laissa fasciner par Dada. Le second groupe se réunit autour de la revue Contemporáneos (1928-1931) qui introduisit le surréalisme au Mexique à travers différents articles, notamment celui de J. Torres Bodet, "Nadja, de André Breton" (No 5. Octobre 1928) et celui de Jorge Cuesta "La poesía de Paul Eluard" (No 12. Mai 1929) suivi de la traduction de 5 poémes de Paul Eluard, du recueil Répétitions (1922), et d'un poéme de Capitale de la douleur (1926). Il est plausible de supposer que la traduction fut également réalisée par Jorge Cuesta. Quelques mois plus tard (No 18 Novembre) il écrivit dans cette même revue un article sur Desnos et le surréalisme.

Letras de México, "Gaceta Literaria y Artística" parut en 1937 et eut une publication mensuelle jusqu'en 1947; cette revue fut dirigée par Barreda, en collaboration avec Villaurrutia, Octavio Paz, Cesar Moro. D’un trés grand intérêt pour nous est le numéro 27 (Mai 1938), car ce numéro spécial fut entièrement consacré à Breton et au surréalisme, avec dès la première page une brève introduction au surréalisme reprenant une déclaration de Marcel Raymond "El sobrerrealismo representa la más reciente tentativa para romper con las cosas que son y para sustituirlas por otras en plena activiad, en plena génesis, cuyos contornos móviles se inscriben en filigrana en el fondo del ser" ainsi qu'une présentation de Breton: "Letras de Mexico se complace en dedicar algunas 
páginas al gran poeta francés. El lector encontrará poesías y textos de prosa de André Breton, poesías y reproducciones de obras plásticas de otros poetas y pintores sobrerrealistas, una bibliografía completa de André Breton y una lista bibliográfica de obras acerca del "movimiento surrealista" "Parallèlement à cette présentation se trouve l'article théorique de Breton "Lo maravilloso contra el misterio" publié dans Minotaure No 9 en 1936 et "Cartero Cheval" et "El gran socorro mortífero" extraits du recueil Le revolver à cheveux blancs (1932) et traduits par César Moro, ainsi que "Un hombre y una mujer absolutamente blancos", appartenant au même recueil et traduit par Xavier Villaurrutia. Quant à la traduction de L'Union Libre elle est due à la plume de Agustín Lazo. La page suivante contient des poèmes de différents surréalistes, principalement Eluard: "La enamorada" (traduction de Villaurrutia), "El Universo-Soledad" (traduction de Wetsphalen), "Entre otras" (traduction de César Moro) ${ }^{6}$. Moro élabora également la traduction d'un poème de Benjamin Péret, "Mil veces"7 et d'un texte en prose de Gui Rosey.

Puis dans les pages suivantes apparaissent un extrait de Les Vases Communicants et l'article "El surrealismo y la pintura" de André Breton.

De la même façon le silence semble être gardé sur la revue Poesía qui n'eut que trois numéros au cours de l'année 1938, numéros auxquels collaborèrent Emilio Prados, Xavier Villaurrutia, Octavio Paz... Cette revue consacra le supplément du No 3 aux surréalistes français et la présentation en fut confiée á César Moro:

"El Surrealismo es el cordón que une la bonba de dinamita con el fuego para hacer la montaña. La cita de las tormentas portadoras del rayo y de la lluvia de fuego. El bosque virgen y la miriada de aves de plumaje eléctrico cubriendo el cielo tempestuoso. La esmeralda de Nerón. Una llanura inmensa poblada de sarcófagos de hielo encerrando lianas y lámparas de acetileno, globos de azogue, mujeres desnudas coronadas de cardos y de fresas. El tigre real qu asola las tierras de tesoros. La estatua de la noche de plumas de paraíso salpicada con sangre de jirafas degolladas bajo la luna. El día inmenso de cristal de roca y los jardines del cristal de roca. Los nombres de SADE, LAUTREAMONT, RIMBAUD, JARY (sic), en formas diversas y delirantes de aerolito sobre una sábana de sangre transparente que agita el viento nocturno sobre el basalto ardiente del imsomnio (sic)"

Suivent des textes de Chirico ("Una noche"), Arp (Configuración"), Breton ("En tu lugar desconfiaría del caballo de paja"), Eluard ("Algunas de las palabras que, hasta ahora, me estaban misteriosamente prohibidas"), Dalí ("El fenómeno biológico"), Duchamp ("Entre nuestros artículos de quincallería"), Hugnet ("La hora del Pastor"), Paalen ("gruta de Bronce"), Péret ("Háblame”), Prassinos ("Anuncios") et Rosey ("He aquí todos los siglos pasados a filo de espada"), tous traduits par César Moro.

(6) Ces poèmes sont extraits de Mourir de ne pas mourir (1924) et La vie Immédiate (1932) (7) extrait de Derrière les fagots (1934)
Cette présence du surréalisme français au Mexique se vit renforcée par le séjour qu'y réalisa Breton en 1938, invité par le gouvernement pour un cycle de conférences. S'y ajoute le long séjour de Péret lors de la Seconde Guerre Mondiale. Il y resta en effect de 1941 à 1948 , ce qui permit sans doute une pénétration plus importante du surréalisme français.

Aprés un séjour en Europe, Octavio Paz revint au Mexique en 1938, imprégné des mouvements 1941 et se chargea de publier des poèmes d'auteurs mexicains et de pòtes espagnols exilés. chargea de publier des poemes d'auteurs "le surréalisme dans toute sa rigueur Bien que d'apres Sarane Alexandrian ${ }^{8}$ "le surréalisme dans toute sa rigueur théorique, et toute la complexité de ses problémes, ne commence à être représenté qu'avec le groupe Taller (1938-1941)" la revue n'inclut dans ses différents numéros ni textes théoriques ni traductions.

Le même phénomène d'une synthèse du national et de l'universel se reproduisi dans Fl Hijo Pródigo, dès 1943 . La revue était divisée en quatre section "Tiempo, Destiempo, Contratiempo y Pasatiempo". Francisco Caudet nous renvoie aux précisions que donna Barreda, directeur de la revue: "En la primera (Tiempo) se incluirán los ensayos, narraciones, teatro y poemas de escritores (Destiempo), las traducciones y los textos del pasado poco conocidos u olvidados; en la tercera (Contratiempo) entrarían las notas críticas -en ocasiones amargas- sobre libros recientes; y en la cuarta (Pastiempo o Entretiempo) todo aquello de por sí fugaz, agudo o bien de mero divertimiento"9.

La revue se voulait ouverte aux poètes et critiques étrangers et publia bon Lombre de traductions de Saint John Perse, Rimbaud, Lautréamont... Pour ce qui est du surréalisme il existe dans le No 14 du mois de Mai 1944 un long article de Péret intitulé "Los Mitos" (traduit par César Moro) où le poète présente une sorte d'anthologie et d'analyse du mythe comme production poétique dans le cadre de la littérature primitive et populaire des peuples américains, pour finalement déboucher une analyse du "merveilleux" et particuliérement du "merveilleux" surréaliste Par la suite dans le No 38 du mois Mai 1946 apparaissent des traductions de poemes de Benjamin Péret (toujours par César Moro) ${ }^{10}$.

\section{Pérou}

Il est difficile de penser que le Pérou ait pu posséder une ambiance favorable au surréalisme, étant donné les circonstances spéciales qui entouraient l'activité littéraire de ce pays, "largas épocas de dictadura militar, censura de la imprenta, aislamiento cultural, obligando mucho de los escritores a buscar el camino del

(8) Alexandrian, Sarane. Le surréalisme et le rêve. Paris. Gallimard. 1974. p. 230.

(9) El Hijo Pródigo. Antología. Introducción, selección y notas de Francisco Caudet. Mexico. Siglo XXI Editores. 1979. p. 16.

(10) "Para parts (1934) ainsi que "Parpadeo" et "Allo" extraits de Je Sublime (1936). 
exilio o a esconderse en el exilio interior"11. Malgré l'opposition et le rejet total que manifesta un grand poète tel que César Vallejo envers le mouvement surréaliste en publiant dès 1930 "Autopsia del surrealismo" oú il dénonçait l'état de désintégration du mouvement et s'attaquait principalement à Breton, deux hommes vont cependant rendre possible cette percée du surréalisme français au Pérou; César Moro qui revint aprés avoir vécu huit ans à Paris où il avait collaboré à la revue Le Surréalisme au Service de la Révolution; l'autre étant E. Westphalen. Ils réussirent à éditer en Décembre 1939 El Uso de la Palabra, revue ouverte sur l'étranger, oú écrivent A. Lazo, A. Paalen, auxquels s'ajoutent Breton et Eluard ${ }^{12}$. Il nous a été impossible de consulter l'unique numéro de cette revue et nous ignorons donc quels sont les textes ou les traductions qui futent publiés. Par la suite Westphalen créa Las Moradas (1947-49), revue para-suréaliste, á laquelle collabora également César Moro. Dés le premier numéro (1947) nous trouvons des traductions de poémes de Roberto Desnos "Tal como una mano en el instante de la muerte", "A favor de la noche" correspondants à Poèmes à la Mystérieuse.

Le réseau de relations et de connexions existant entre la France et l'Amérique Latine permit la diffusion du surréalisme, non seulement sous forme de références ou échos, mais à travers des textes concrets, théoriques et poétiques, s'identifiant au projet du groupe formé autour d'André Breton. La plupart des groupes d'avant-garde faisait donc clairement référence au surréalisme français, même si certains ne faisaient que revendiquer leur dette envers ce mouvement. Les différentes traductions mentionnées et les différents textes théoriques ont pour auteurs les grands chefs de file du mouvement (Breton, Péret, Eluard et dans une moindre mesure Desnos). On ne trouve les auteurs mineurs que dans le supplément de Poesía en 1938. Il s'agit dans l'ensemble de textes appartenant à la "derniére" époque du mouvement, textes qui étaient contemporains des traducteurs et qui présentent non pas le surréalisme hésitant des premiers temps mais un surréalisme qui domine parfaitement ses techniques de prospection de la vie et de la poésie.

(11) Baciu, Stefan Op. Cit. p. 111.

(12) selon l'affirmation de Stefan Baciu Op. Cit. p. 114 et Pierre Rivas Op. Cit. p. 109-114. 